\title{
On Projective Modules and Computation of Dimension of a Module over Laurent Polynomial Ring
}

\author{
Ratnesh Kumar Mishra, Shiv Datt Kumar, and Srinivas Behara \\ Department of Mathematics, Motilal Nehru National Institute of Technology, Allahabad 211004, India \\ Correspondence should be addressed to Ratnesh Kumar Mishra, rkmishra814@gmail.com
}

Received 18 April 2011; Accepted 9 May 2011

Academic Editors: A. Fialowski and A.-G. Wu

Copyright (C) 2011 Ratnesh Kumar Mishra et al. This is an open access article distributed under the Creative Commons Attribution License, which permits unrestricted use, distribution, and reproduction in any medium, provided the original work is properly cited.

We give a procedure and describe an algorithm to compute the dimension of a module over Laurent polynomial ring. We prove the cancellation theorems for projective modules and also prove the qualitative version of Laurent polynomial analogue of Horrocks' Theorem.

\section{Introduction}

Hilbert [1] introduced free resolution on iterated syzygies of a finitely generated graded module $M$ over polynomial ring $R=K\left[x_{1}, \ldots, x_{n}\right]$. A choice of $a_{0}=\operatorname{dim}\left(M \otimes_{R} K\right)$ homogeneous generators of $M$ defines a surjective homomorphism $R^{a_{0}} \rightarrow M$, and its kernel is the first syzygy module of $M$. Hilbert proved that the syzygies are also finitely generated (Hilbert basis theorem). Hilbert's syzygy theorem states that "every finitely generated module $M$ over $K\left[x_{1}, \ldots, x_{n}\right]$ has finite free resolution, that is, the $l$ th syzygy is $R^{a_{l}}$ for some $a_{l} . "$ The number of generators $a_{i}$ of the syzygies is chosen minimally, they are independent of the choice of generators. These $a_{i}$ are called the Betti numbers of $M$ and the minimal length of free resolution (i.e., $i$ ) is dimension of a module $M$. When $M$ is projective, the dimension of $M$ is called projective dimension of $M$ (denoted by $\operatorname{pd}(M)$ ). A projective dimension is a measure of how far the module is being projective.

Kaplansky [2] described how homological dimension changes when one passes from a ring $R$ to a quotient ring $R /(X)$, where $(X)$ is the ideal generated by a nonzero divisor, without using Ext or Tor. Shanuel noticed that there is an elegant relation between different projective resolutions of the same module. Using the ideas of Shanuel, Kaplansky defined the projective dimension of a module. 
The global dimension (or homological dimension) of a ring $R$ denoted by gldim $(R)$, is a non-negative integer or infinity, and is a homological invariant of the ring. It is defined to be the supremum of the set of projective dimensions of all (finite or cyclic) $R$-modules. Global dimension is an important technical notion in the dimension theory of Noetherian rings. Eisenbud [3] proved that a commutative Noetherian local ring $R$ is regular if and only if it has finite global dimension, in which case the global dimension coincides with the Krull dimension of $R$. This theorem opened the door for application of homological methods to commutative algebra. Now we state a theorem of Eisenbud [3] which implies that every module has a finite free resolution of length at most $l<\infty$.

Theorem 1.1 (see [3]). The following conditions on a ring $R$ are equivalent:

(1) $\operatorname{gldim}(R) \leq l$, that is, $\operatorname{pd}(M) \leq l$ for every $R$-module $M$,

(2) $\operatorname{pd}(M) \leq l$ for every finitely generated module $M$.

Serre [4] taught a course on multiplicities at the college de France. Part of that course focussed upon the simple inequality $\operatorname{pd}_{R}(M) \leq \operatorname{pd}_{R}(S)+\operatorname{pd}_{S}(M)$ for module $M$ over an $R$-algebra $S$. Auslander and Buchsbaum [5] realized that Serre's method could be used to study the close connection between the dimension and multiplicity over a local ring. This led them to the Auslander-Buchsbaum equality [6]: let $M$ be a finitely generated module over a Noetherian local ring $(R, m)$. If $\operatorname{pd}(M)<\infty$, then $\operatorname{depth}(R)=\operatorname{depth}(M)+\operatorname{pd}(M)$. GagoVargas [7] computed the projective dimension of a module over Weyl algebra. Projective dimension is a main ingredient to compute the tilted algebra which nowadays plays a very important role in the representation theory of algebras. In this paper, we give a procedure and describe an algorithm to compute the projective dimension of a module which is valid over the Laurent polynomial ring.

We also prove the cancellation theorems for projective modules over the Laurent polynomial ring [8, Section 5] motivated by Swan's work. Horrocks proved the following theorem.

Theorem 1.2 (see [9]). Let $R$ be a local ring, $A=R[x]$ and $S$ be the set of monic polynomials of $A$. If $P$ is a finitely generated projective $A$-module such that $P_{S}$ is free over $A_{S}$, then $P$ is a free A-module.

This theorem draw the attention of several mathematicians, as a result several analogues of Horrocks' theorem can be found in the literature [10,11]. Mandal proved the Laurent polynomial version of Horrocks' theorem in [12] whereas Nashier [13] proved the qualitative version of the same theorem over polynomial ring. But it is observed that the qualitative version of Horrocks' theorem over Laurent polynomial ring is not found anywhere in the literature. One of the objectives of this paper is to fill this gap. In this paper, we prove the qualitative version of Horrocks' theorem over the Laurent polynomial ring.

\section{Preliminary Notes}

In this section we define some terms used in this paper and state certain standard results without proof. We hope that this will improve the readability and understanding of the proof of the paper.

A polynomial $f$ in the Laurent polynomial ring $R\left[X, X^{-1}\right]$ is said to be doubly monic polynomial if coefficient of the highest degree term and the lowest degree term are unit. 

Section 3

We need the following result of Kunz [14] to compute the projective resolution in

Definition 2.1 (see [14]). An exact sequence

$$
F_{i+1} \stackrel{\alpha_{i}}{\longrightarrow} F_{i} F_{1} \stackrel{\alpha_{0}}{\longrightarrow} F_{0} \stackrel{\epsilon}{\longrightarrow} M \rightarrow 0
$$

with only free (resp., projective) modules $F_{i}(i=0,1,2, \ldots)$ is called a free (resp., projective) resolution of $M$.

Projective modules over the Laurent polynomial ring $R\left[X, X^{-1}\right]$ are stably free. In [15] it is proved that a projective module is stably free provided it possesses a finite free resolution. The minimum length of free resolution is called the projective dimension of a module $M$.

Let $R$ be a ring and $M$ be an $R$-module. $M\left[X, X^{-1}\right]$ means the analogously defined Laurent polynomial module. We identify $M \otimes_{R} R\left[X, X^{-1}\right]$ with $M\left[X, X^{-1}\right]$, where $M\left[X, X^{-1}\right]=$ $\left\{\sum_{n=-\infty}^{\infty} m_{n} X^{n} \mid m_{n} \in M\right.$ and $m_{n}=0$ for all but finite $\left.n\right\}$. If $f: M \rightarrow N$ is a homomorphism of $R$-modules, then this induces a homomorphism

$$
\begin{array}{r}
\psi: M\left[X, X^{-1}\right] \rightarrow N\left[X, X^{-1}\right], \\
\text { defined by } \psi\left(\sum m_{n} X^{n}\right)=\sum f\left(m_{n}\right) X^{n},
\end{array}
$$

where $\psi$ can be identified with $f \otimes_{R} R\left[X, X^{-1}\right]$. Given any $R$-module $M$ and $f \in \operatorname{End}_{R}(M)$, we can make $M$ as $R\left[X, X^{-1}\right]$-module whose scalar multiplication is defined as $m\left(\sum a_{n} X^{n}\right)=$ $a_{n} \sum f^{n}(m)$. We denote this $R\left[X, X^{-1}\right]$-module by ${ }_{f} M$. There is a canonical $R\left[X, X^{-1}\right]$ epimorphism $\varphi_{f}: M\left[X, X^{-1}\right] \rightarrow{ }_{f} M$ defined by $\varphi_{f}\left(\sum m_{n} X^{n}\right)=\sum f^{n}\left(m_{n}\right)$. The characteristic sequence of $f$ is an exact sequence which proved in Proposition 4.3.

The following is the Laurent polynomial version of a Horrocks Theorem which we state as follows.

Theorem 2.2 (see [12]). Suppose $R\left[X, X^{-1}\right]$ is a Laurent polynomial ring over a local Noetherian commutative ring $R$, and $P$ is a projective $R\left[X, X^{-1}\right]$-module. If $P_{f}$ is free for some doubly monic Laurent polynomial $f$, then $P$ is free.

In the proof of the Theorem 5.1, we need the following theorem.

Theorem 2.3 (see [12]). Suppose that $R\left[X, X^{-1}\right]$ is a Laurent polynomial ring over a local Noetherian commutative ring $R$. Also suppose that $P$ and $P^{\prime}$ are two projective $R\left[X, X^{-1}\right]$-modules with Rank $P^{\prime}<\operatorname{Rank} P$. If $P_{f}^{\prime}$ is a direct summand of $P_{f}$ for some doubly monic polynomial $f$, then $P^{\prime}$ is also a direct summand of $P$.

\section{Projective Dimension of Module}

Lemma 3.1. Let $M_{1}, M_{2}, M_{3}$ be R-modules and

$$
0 \longrightarrow M_{1} \stackrel{\alpha_{1}}{\longrightarrow} M_{2} \stackrel{\alpha_{2}}{\longrightarrow} M_{3} \longrightarrow 0
$$


be a split short exact sequence, and $\beta_{1}$ and $\beta_{2}$ are the splittings corresponding to $\alpha_{1}$ and $\alpha_{2}$, respectively. Then the following sequence

$$
0 \longrightarrow M_{3} \stackrel{\beta_{2}}{\longrightarrow} M_{2} \stackrel{\beta_{1}}{\longrightarrow} M_{1} \longrightarrow 0
$$

is an exact sequence.

Proof. Since $\beta_{1}$ and $\beta_{2}$ are the splittings corresponding to $\alpha_{1}$ and $\alpha_{2}$, respectively, then $\beta_{1} O \alpha_{1}=$ $I_{M_{1}}$ and $\alpha_{2} O \beta_{2}=I_{M_{3}}$. Let $x \in M_{2}$. Then $\alpha_{2}\left(x-\beta_{2}\left(\alpha_{2}(x)\right)\right)=\alpha_{2}(x)-\alpha_{2}\left(\beta_{2}\left(\alpha_{2}(x)\right)\right)=\alpha_{2}(x)-$ $\alpha_{2}(x)=0$. Hence $x-\beta_{2}\left(\alpha_{2}(x)\right) \in \operatorname{Ker}\left(\alpha_{2}\right)=\operatorname{Im}\left(\alpha_{1}\right)$. Since $\alpha_{1}$ is injective, there exists a unique $\beta_{1}(x) \in M_{1}$ such that $\alpha_{1}\left(\beta_{1}(x)\right)=x-\beta_{2}\left(\alpha_{2}(x)\right)$. We need to show that $\operatorname{Ker}\left(\beta_{1}\right) \subset \operatorname{Im}\left(\beta_{2}\right)$. Let $x \in \operatorname{Ker}\left(\beta_{1}\right)$. Then $\beta_{1}(x)=0$ and $\alpha_{1}\left(\beta_{1}(x)\right)=\alpha_{1}(0)=0$, by injectivity of $\alpha_{1}$. Hence $x-\beta_{2}\left(\alpha_{2}(x)\right)=0 \Rightarrow x=\beta_{2}\left(\alpha_{2}(x)\right)$. Thus there exists $\alpha_{2}(x) \in M_{3}$ such that $\beta_{2}\left(\alpha_{2}(x)\right)=x$. Hence $x \in \operatorname{Im}\left(\beta_{2}\right)$. Therefore $\operatorname{Ker} \beta_{1} \subset \operatorname{Im}\left(\beta_{2}\right)$. Conversely, let $x \in \operatorname{Im}\left(\beta_{2}\right)$. Then there exists $y \in M_{3}$ such that $\beta_{2}(y)=x$. Applying $\alpha_{2}$ on both sides, we get $y=\alpha_{2}(x)$. Now applying $\beta_{2}$ on both sides, we get $\beta_{2} \alpha_{2}(x)=x$ and therefore $x-\beta_{2} \alpha_{2}(x)=0$. Using the defining property of $\beta_{1}$ and injectivity of $\alpha_{1}, \alpha_{1}\left(\beta_{1}(x)\right)=0=\alpha_{1}(0)$. Therefore $\beta_{1}(x)=0$. Hence $x \in \operatorname{Ker}\left(\beta_{1}\right)$. Thus $\operatorname{Im}\left(\beta_{2}\right) \subset \operatorname{Ker}\left(\beta_{1}\right)$. Therefore $\operatorname{Im}\left(\beta_{2}\right)=\operatorname{Ker}\left(\beta_{1}\right)$

By applying the technique discussed in [7] for Weyl algebra, we give a procedure to calculate the projective dimension of a module over the Laurent polynomial ring. We also describe an algorithm to compute the projective dimension of a module.

Procedure. The first step is to define the Laurent polynomial ring $R\left[x_{1}^{ \pm 1}, x_{2}^{ \pm 1}, \ldots, x_{n}^{ \pm 1}\right]$ in terms of quotient ring as

$$
R\left[x_{1}^{ \pm 1}, x_{2}^{ \pm 1}, \ldots, x_{n}^{ \pm 1}\right] \cong \frac{R\left[x_{1}, y_{1}, x_{2}, y_{2}, \ldots, x_{n}, y_{n}\right]}{\left(x_{1} y_{1}-1, x_{2} y_{2}-1, \ldots, x_{n} y_{n}-1\right)}
$$

The second step is to test whether the $R\left[x_{1}^{ \pm 1}, \ldots, x_{n}^{ \pm 1}\right]$-module $M$ is projective or not. If $M$ is projective, then again we test whether $M$ is stably free or not, that is, we find a matrix that defines an isomorphism $M \oplus R\left[x_{1}^{ \pm 1}, \ldots, x_{n}^{ \pm 1}\right]^{r} \cong R\left[x_{1}^{ \pm 1}, \ldots, x_{n}^{ \pm 1}\right]^{s}$, for some positive integers $r$ and $s$. If $M$ is stably free, then the next step is to find finite free resolution of the projective module $M$. We denote the homomorphisms with their matrices to simplify the notation.

Given an $R\left[x_{1}^{ \pm 1}, \ldots, x_{n}^{ \pm 1}\right]$-module $M$ defined by a system of generators in some $R\left[x_{1}^{ \pm 1}, \ldots, x_{n}^{ \pm 1}\right]^{t}$, choose a free $R\left[x_{1}^{ \pm 1}, \ldots, x_{n}^{ \pm 1}\right]$-module $P_{0}$ and a surjection $\sigma: P_{0} \rightarrow M$ with kernel $C_{0}$, then we get an exact sequence

$$
0 \longrightarrow C_{0} \stackrel{\psi_{0}}{\longrightarrow} P_{0} \stackrel{\sigma}{\longrightarrow} M \longrightarrow 0
$$

Now, choose a free $R\left[x_{1}^{ \pm 1}, \ldots, x_{n}^{ \pm 1}\right]$-module $P_{1}$ together with a surjection map $\phi_{1}: P_{1} \rightarrow C_{0}$ with kernel $C_{1}$. Again we choose a free $R\left[x_{1}^{ \pm 1}, \ldots, x_{n}^{ \pm 1}\right]$-module $P_{2}$ together with a surjection $\phi_{2}: P_{2} \rightarrow C_{1}$ with kernel $C_{2}$, then we get an exact sequence

$$
0 \longrightarrow C_{2} \stackrel{\psi_{2}}{\longrightarrow} P_{2} \stackrel{\phi_{2}}{\longrightarrow} C_{1} \stackrel{\psi_{1}}{\longrightarrow} P_{1} \stackrel{\phi_{1}}{\longrightarrow} C_{0} \stackrel{\psi_{0}}{\longrightarrow} P_{0} \stackrel{\sigma=\alpha_{0}}{\longrightarrow} M \longrightarrow 0
$$


Continuing this process, we have an exact sequence

$$
\wp: 0 P_{l} \stackrel{\alpha_{l}}{\longrightarrow} P_{l-1} \stackrel{\alpha_{l-1}}{\longrightarrow} P_{1} \stackrel{\alpha_{1}}{\longrightarrow} P_{0} \stackrel{\sigma=\alpha_{0}}{\longrightarrow} M \longrightarrow 0
$$

which is a free resolution of the module $M$, with $\operatorname{rank}\left(P_{i}\right)=t_{i}$, where $\alpha_{l}=\psi_{l-1} \phi_{l}$. For $l=1$

$$
0 \longrightarrow P_{1} \stackrel{\alpha_{1}}{\longrightarrow} P_{0} \stackrel{\alpha_{0}}{\longrightarrow} M \longrightarrow 0
$$

is a free resolution of module $M$. Since $M$ is a projective module, this sequence splits, so there exists $\beta_{1}: P_{0} \rightarrow P_{1}$ such that $\beta_{1} \alpha_{1}=I_{t_{1}}$. We can compute the matrix $\beta_{1}$ from the rows of the matrix $\alpha_{1}$. We express each vector of the canonical basis of $P_{1}$ as a linear combination of the rows of $\alpha_{1}$, and with these coefficients we construct the matrix $\beta_{1}$. Using Lemma 3.1, the exact sequence in (3.7) splits, giving another exact sequence

$$
0 \longrightarrow M \stackrel{\beta_{0}}{\longrightarrow} P_{0} \stackrel{\beta_{1}}{\longrightarrow} P_{1} \longrightarrow 0
$$

Then $P_{1} \oplus M \cong P_{0} \cong \operatorname{Ker}\left(\beta_{1}\right) \oplus P_{1}$. Since $M$ is a projective module, the short exact sequence

$$
0 \longrightarrow \operatorname{Ker}\left(\alpha_{0}\right) \longrightarrow P_{0} \stackrel{\alpha_{0}}{\longrightarrow} M \longrightarrow 0
$$

splits, so $\operatorname{Ker}\left(\alpha_{0}\right)=\operatorname{Im}\left(\alpha_{1}\right)$ is projective. Hence $\operatorname{pd}(M)=0$. Suppose $M$ is not projective, then the short exact sequence (3.9) does not split. Therefore $\operatorname{Im}\left(\alpha_{1}\right)=\operatorname{Ker}\left(\alpha_{0}\right)$ is not projective. For $l=2$, the exact sequence (3.6) does not split, therefore $\operatorname{Im}\left(\alpha_{2}\right)=\operatorname{Ker}\left(\alpha_{1}\right)$ is not projective. Continuing this process $\operatorname{Im}\left(\alpha_{1}\right), \operatorname{Im}\left(\alpha_{2}\right), \ldots, \operatorname{Im}\left(\alpha_{l-1}\right)$ are not projective and $\operatorname{Im}\left(\alpha_{l}\right)$ is projective. In this way after performing a finite number of steps, we obtain the minimum length of free resolution of $M$ which is the projective dimension of $M$ such that $\operatorname{pd}(M)=l$ see Algorithm 1.

Remark 3.2. Now we give an algorithm to calculate the projective dimension of a module over the Laurent polynomial ring. We follow the technique discussed in [16] over polynomial ring.

Example 3.3. Let

$$
\begin{aligned}
& \qquad A=R\left[x, x^{-1}, y, y^{-1}\right] \cong \frac{R[x, y, p, q]}{(x p-1, y q-1)} \\
& \operatorname{ring} R=0,(x, y, p, q), d p ; \\
& \operatorname{ideal~} I=x p-1, y q-1 ; \\
& I=\operatorname{std}(I) ; \\
& I ; \\
& I[1]=y q-1 ;
\end{aligned}
$$


Let

$$
A=R\left[x_{1}^{ \pm 1}, x_{2}^{ \pm 1}, \ldots, x_{n}^{ \pm 1}\right] \cong \frac{R\left[x_{1}, y_{1}, x_{2}, y_{2}, \ldots, x_{n}, y_{n}\right]}{\left(x_{1} y_{1}-1, x_{2} y_{2}-1, \ldots, x_{n} y_{n}-1\right)} .
$$

Objective: Computation of the projective dimension of a module. Input: A finitely generated $A$-module $M=<f_{1}, f_{2}, \ldots, f_{m}>\subset A^{t}$ and a positive integer $l$.

Output: A projective dimension of $M$ and a list of matrices $\alpha_{1}, \alpha_{2}, \ldots, \alpha_{l}$ with $\alpha_{i} \in \operatorname{Mat}\left(l_{i-1} \times l_{i}, A\right)$, where $i=1,2, \ldots, l$, such that

$$
\text { }: 0 P_{1} \stackrel{\alpha_{l}}{\longrightarrow} P_{l-1} \stackrel{\alpha_{l-1}}{\longrightarrow} P_{1} \stackrel{\alpha_{1}}{\longrightarrow} P_{0} \stackrel{\alpha_{0}}{\longrightarrow} P_{0} / M \longrightarrow 0
$$

is a free resolution of $P_{0} / M$. If $\operatorname{pd}(M)=0$, then $M$ is projective. The algorithm returns $M \oplus A^{r} \cong A^{l}$.

START:

initialize $i=1$;

if ( $\alpha_{1}$ does not split)

$\alpha_{1}=\operatorname{matrix}\left(f_{1}, f_{2}, \ldots, f_{m}\right) \in \operatorname{Mat}(l \times m, A)$ and $\operatorname{pd}(M)=1$;

else

Let $\beta_{1}$ be the split of $\alpha_{1}$. Then $\operatorname{pd}(M)=0$ and

$P_{1} \oplus M \cong P_{0} \cong \operatorname{ker}\left(\beta_{1}\right) \oplus P_{1} ;$

end if

if $(i=l)$

return $\operatorname{pd}(M)$;

else

while $(i<l)$ do

$i++$;

$\alpha_{i}=\operatorname{syz}\left(\alpha_{i-1}\right)$;

end loop

end if

if $\left(\alpha_{l}\right.$ does not split)

$\operatorname{pd}(M)=l$;

else

Let $\beta_{l}$ be the split of $\alpha_{l}$. Then $\operatorname{pd}(M)=l-1$;

end if

return $\operatorname{pd}(M)$;

STOP.

\section{Algorithm 1}

$I[2]=x p-1$;

qring $A=I$;

A;

${ }^{* *}$ quotient ring from ideal ${ }^{* *}$

[1] = yq-1;

[2] $=x p-1$;

Poly $f_{1}=x^{3} p+\left(x^{3} p\right)^{-1}+y^{4} q$;

Poly $f_{2}=x p+(y q)^{-1}+y q$;

Ideal $M=\left(f_{1}, f_{2}\right)$;

Resolution $L=\operatorname{mres}(M, 0)$; 
We find a resolution of $P_{0} / M$ over Laurent polynomial ring:

$$
0 \rightarrow P_{0} \stackrel{\alpha_{0}}{\rightarrow} P_{0} / M \rightarrow 0
$$

Hence, projective dimension of $M$ is 0 .

Example 3.4. Let

$$
\begin{aligned}
A & =R\left[x, x^{-1}, y, y^{-1}, z, z^{-1}, s, s^{-1}\right] \\
& \cong \frac{R[x, y, z, p, q, u, s, t]}{(x p-1, y q-1, z u-1, s t-1)}
\end{aligned}
$$

$$
\begin{aligned}
& \operatorname{ring} R=0,(x, y, z, p, q, u, s, t), d p ; \\
& \text { ideal } I=x p-1, y q-1, z u-1, s t-1 ; \\
& I=\operatorname{std}(I) ; \\
& I ; \\
& I[1]=s t-1 \\
& I[2]=z u-1 \\
& I[3]=y q-1 \\
& I[4]=x p-1 \\
& \text { qring } A=I ;
\end{aligned}
$$$$
\text { ideal } I=x p-1, y q-1, z u-1, s t-1 \text {; }
$$

A;

${ }^{* *}$ quotient ring from ideal ${ }^{* *}$

$$
\begin{aligned}
& {[1]=s t-1} \\
& {[2]=z u-1} \\
& {[3]=y q-1} \\
& {[4]=x p-1} \\
& \text { poly } f=x^{3} p+\left(x^{3} p\right)^{-1}+y^{4} q+z u^{2} ; \\
& \text { poly } g=x^{6} p^{2}+(y q)^{-1}+y q+s^{3} t+y q ; \\
& \text { ideal } M=(f, g) ; \\
& \text { resolution } L=\operatorname{mres}(M, 0) .
\end{aligned}
$$

We find a resolution of $P_{0} / M$ over Laurent polynomial ring:

$0 \rightarrow P_{32}^{3} \oplus P_{33}^{6} \stackrel{\alpha_{11}}{\longrightarrow} P_{30} \oplus P_{31}^{5} \stackrel{\alpha_{10}}{\longrightarrow} P_{28}^{2} \oplus P_{29}^{3} \stackrel{\alpha_{9}}{\longrightarrow} P_{26}^{2} \oplus P_{27}^{2} \stackrel{\alpha_{8}}{\longrightarrow} P_{20}^{-1} \oplus P_{21} \oplus P_{22}^{3} \oplus P_{23} \oplus P_{24} \oplus P_{25} \stackrel{\alpha_{7}}{\longrightarrow}$ $P_{15}^{2} \oplus P_{16}^{2} \oplus P_{17}^{2} \oplus P_{18} \oplus P_{19} \stackrel{\alpha_{6}}{\longrightarrow} P_{11}^{3} \oplus P_{12}^{2} \oplus P_{13}^{2} \oplus P_{14} \stackrel{\alpha_{5}}{\longrightarrow} P_{8}^{4} \oplus P_{9}^{2} \oplus P_{10}^{2} \stackrel{\alpha_{4}}{\rightarrow} P_{5}^{3} \oplus P_{6} \oplus P_{7} \stackrel{\alpha_{3}}{\longrightarrow} P_{2}^{-1} \oplus P_{3} \oplus P_{4} \stackrel{\alpha_{2}}{\longrightarrow}$ $P_{1} \stackrel{\alpha_{1}}{\longrightarrow} P_{0}^{0} \stackrel{\alpha_{0}}{\longrightarrow} P_{0} / M \rightarrow 0$.

Hence, projective dimension of $M$ is 11 .

Remark 3.5. Examples 3.3 and 3.4 are verified using the singular software [17]. 


\section{A Cancellation Theorem for Projective Modules over Laurent Polynomial Ring}

Proposition 4.1. Let $P$ and $P^{\prime}$ be projective $R\left[X, X^{-1}\right]$-modules and let $\varphi: P^{\prime} \rightarrow P, \psi: P \rightarrow P^{\prime}$ be injective homomorphisms. If $R\left[X, X^{-1}\right], P / \varphi \psi P$, and $P^{\prime} / \psi \varphi P^{\prime}$ are projective over $R$, then $P / \varphi P^{\prime}$ and $P^{\prime} / \psi P$ are also projective over $R$.

Proof. Since $P$ and $P^{\prime}$ are $R\left[X, X^{-1}\right]$-projective and $R\left[X, X^{-1}\right]$ is $R$-projective, then we have

$$
\begin{gathered}
P \oplus P_{1} \cong R\left[X, X^{-1}\right]^{n} \\
P^{\prime} \oplus P_{1}^{\prime} \cong R\left[X, X^{-1}\right]^{m} \\
R\left[X, X^{-1}\right] \oplus Q \cong \sum R \\
\vdots \\
R\left[X, X^{-1}\right]^{n} \oplus Q^{n} \cong \sum R \\
P \oplus P_{1} \oplus Q^{n} \cong \sum R \\
P \oplus Q_{1}^{n} \cong \sum R,
\end{gathered}
$$

where $P_{1} \oplus Q^{n}=Q_{1}^{n}$. Therefore, $P$ is a $R$-projective. Similarly $P^{\prime}$ is also a $R$-projective. Since

$$
0 \longrightarrow P \stackrel{\beta}{\longrightarrow} P^{\prime} \longrightarrow P^{\prime} / \psi P \longrightarrow 0
$$

$\operatorname{pd}\left(P^{\prime} / \psi P\right) \leq 1$ and similarly $\operatorname{pd}\left(P / \varphi P^{\prime}\right) \leq 1$. Now isomorphism between $P$ and $\psi P$ induces $P / \varphi P^{\prime} \cong \psi P / \psi \varphi P^{\prime}$. We have the exact sequences

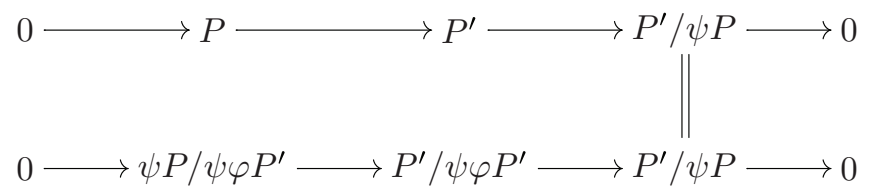

By Schanuel's lemma, $P^{\prime} / \psi \varphi P^{\prime} \oplus P \cong \psi P / \psi \varphi P^{\prime} \oplus P^{\prime}$ so that $P^{\prime} / \psi \varphi P^{\prime} \oplus P \cong P / \varphi P^{\prime} \oplus P^{\prime}$. Since $P^{\prime} / \psi \varphi P^{\prime}, P$ are $R$-projective and direct sum of projective module is also projective module, then $P^{\prime} / \psi \varphi P^{\prime} \oplus P$ is $R$-projective. By the isomorphism, $P / \varphi P^{\prime} \oplus P^{\prime}$ is an $R$-projective. $P / \varphi P^{\prime} \oplus$ $P^{\prime} \oplus P_{0} \cong R^{n}$ follows from the definition of $R$-projective module, where $P_{0}$ is an $R$-module. Let $\overline{\mathrm{P}}=P^{\prime} \oplus P_{0}$ be an $R$-module. Then $P / \varphi P^{\prime} \oplus \overline{\mathrm{P}} \cong R^{n}$. Hence $P / \varphi P^{\prime}$ is an $R$-projective. Similarly $P^{\prime} / \psi P$ is also an $R$-projective.

Corollary 4.2. Let $P$ and $P^{\prime}$ be projective $R\left[X, X^{-1}\right]$-modules with $P \supset P^{\prime} \supset f P$, where $f$ is a doubly monic polynomial of Laurent polynomial ring. If $R\left[X, X^{-1}\right]$ and $R\left[X, X^{-1}\right] / f R\left[X, X^{-1}\right]$ are $R$-projective, then $P / P^{\prime}$ is also R-projective. 
Proof. Take $\varphi$ the inclusion map $P^{\prime} \hookrightarrow P$ and $\psi$ the multiplication by $f$ from $P \rightarrow P^{\prime}$. Then $P / \varphi \psi P=P / f P$ and $P^{\prime} / \psi \varphi P^{\prime}=P^{\prime} / f P^{\prime}$. Since $P, P^{\prime}$ are $R\left[X, X^{-1}\right]$-projective and $R\left[X, X^{-1}\right]$, $R\left[X, X^{-1}\right] / f R\left[X, X^{-1}\right]$ are $R$-projective. Then $P / f P$ and $P^{\prime} / f P^{\prime}$ are $R\left[X, X^{-1}\right] / f R\left[X, X^{-1}\right]-$ projective. Therefore $P / f P$ and $P^{\prime} / f P^{\prime}$ are $R$-projective. Hence $P / \varphi \psi P$ and $P^{\prime} / \psi \varphi P^{\prime}$ are also $R$-projective. By Proposition 4.1, module $P / P^{\prime}\left(=P / \varphi P^{\prime}\right)$ is $R$-projective.

Proposition 4.3. Let $M$ be an $R$-module and $f \in \operatorname{End}_{R}(M)$. The characteristic sequence of $f$

$$
0 \longrightarrow M\left[X, X^{-1}\right] \stackrel{X \cdot 1_{M\left[X, X^{-1}\right]}-f\left[X, X^{-1}\right]}{\longrightarrow} M\left[X, X^{-1}\right] \stackrel{\varphi_{f}}{\longrightarrow} f M \longrightarrow 0
$$

is an exact sequence in $R\left[X, X^{-1}\right]$-module.

Proof. Clearly $\varphi_{f}$ is surjective, and we have

$$
\begin{aligned}
\varphi_{f}\left(X .1_{M\left[X, X^{-1}\right]} f\left[X, X^{-1}\right]\right)\left(\sum m_{n} X^{n}\right) & =\varphi_{f}\left(\sum\left(m_{n} X^{n+1}-f\left(m_{n}\right) X^{n}\right)\right) \\
& =\sum\left(f^{n+1}\left(m_{n}\right)-f^{n}\left(f\left(m_{n}\right)\right)\right) \\
& =\sum\left(f^{n+1}\left(m_{n}\right)-f^{n+1}\left(m_{n}\right)\right) \\
& =0 .
\end{aligned}
$$

$\operatorname{Im}\left(X .1_{M\left[X, X^{-1}\right]}-f\left[X, X^{-1}\right]\right) \subseteq \operatorname{Ker}\left(\varphi_{f}\right)$. Since, $X .1_{M\left[X, X^{-1}\right]}-f\left[X, X^{-1}\right]$ raises degree by one, and preserves leading coefficients, it is monomorphism. Finally, suppose

$$
z=\sum m_{n} X^{n} \in \operatorname{Ker}\left(\varphi_{f}\right) \Longrightarrow \varphi_{f}\left(\sum m_{n} X^{n}\right)=0, \text { that is, } \sum f^{n}\left(m_{n}\right)=0
$$

Then

$$
\begin{aligned}
& z=z-\sum f^{n}\left(m_{n}\right) \\
&= \sum\left(m_{n} X^{n}-f^{n}\left(m_{n}\right)\right) \\
&=\sum\left(X^{n} .1_{M\left[X, X^{-1}\right]}-f^{n}\right)\left(m_{n}\right) \\
&=\left(X .1_{M\left[X, X^{-1}\right]}-f\left[X, X^{-1}\right]\right)\left[\cdots-\frac{1}{X-f} \frac{X^{n}-f^{n}}{X^{n} f^{n}}-\frac{1}{X-f} \frac{X^{n-1}-f^{n-1}}{X^{n-1} f^{n-1}} \cdots\right. \\
& \quad-\frac{1}{X-f} \frac{X-f}{X f}+0+\frac{X-f}{X-f}+\frac{X^{2}-f^{2}}{X-f}+\cdots \\
&\left.\quad+\frac{X^{n}-f^{n}}{X-f}+\cdots\right]\left(m_{n}\right)
\end{aligned}
$$




$$
\begin{aligned}
& =\left(X \cdot 1_{\mathrm{M}\left[X, X^{-1}\right]}-f\left[X, X^{-1}\right]\right) \sum h_{n}\left(m_{n}\right) \\
\Longrightarrow z & \in \operatorname{Im}\left(X .1_{M\left[X, X^{-1}\right]}-f\left[X, X^{-1}\right]\right) .
\end{aligned}
$$

Therefore $\operatorname{Ker}\left(\varphi_{f}\right) \subseteq \operatorname{Im}\left(X .1_{M\left[X, X^{-1}\right]}-f\left[X, X^{-1}\right]\right)$. Hence $\operatorname{Im}\left(X .1_{M\left[X, X^{-1}\right]}-f\left[X, X^{-1}\right]\right)=\operatorname{Ker}\left(\varphi_{f}\right)$.

Theorem 4.4. Let $P$ and $P^{\prime}$ be finitely generated projective modules over $R\left[X, X^{-1}\right]$. Suppose that $P \supset P^{\prime} \supset f P$ for some doubly monic polynomial $f \in R\left[X, X^{-1}\right]$. Then $P$ and $P^{\prime}$ are stably isomorphic. In particular, if $P_{f} \cong P_{f}^{\prime}$, then $P$ and $P^{\prime}$ are stably isomorphic.

Proof. Take $M=P / P^{\prime}$. Since $f$ is doubly monic polynomial, $R\left[X, X^{-1}\right] /(f)$ is free as an $R$ module. Therefore $P / f P$ is $R$-projective, whence $M$ is $R$-projective by Corollary 4.2 . We have exact sequences of $R\left[X, X^{-1}\right]$-modules

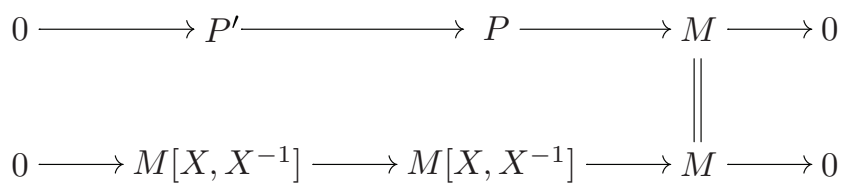

where $M\left[X, X^{-1}\right]=M \otimes_{R} R\left[X, X^{-1}\right]$. Since $P^{\prime} \subset P$ and $M=P / P^{\prime}$, the inclusion map $i$ from $P^{\prime}$ to $P$ and the surjective map $\pi$ from $P$ to $M$ makes the first sequence an exact sequence. By Proposition 4.3, second sequence is also an exact sequence. Since $M$ is an $R$-projective, $M\left[X, X^{-1}\right]$ is an $R\left[X, X^{-1}\right]$-projective. Therefore by the Schanuel's lemma, $P \oplus M\left[X, X^{-1}\right] \cong$ $P^{\prime} \oplus M\left[X, X^{-1}\right]$. Hence $P, P^{\prime}$ are stably isomorphic.

Theorem 4.5. Let $F$ be a free $R\left[X, X^{-1}\right]$-module of $\operatorname{rank} 2, P$ a projective $R\left[X, X^{-1}\right]$-module and $F \supset P \supset X F$. Then $P \cong F$.

Proof. Let $\left\{f_{1}, f_{2}\right\}$ be a free $R\left[X, X^{-1}\right]$-basis for $F$, and let $F_{1}=\left\{a_{1} f_{1}+a_{2} f_{2} \mid a_{1}, a_{2} \in R\right\}$. Then $F_{1}$ is free over $R$ of rank 2 .

Claim. We show that each element of $F_{1} \oplus X F \oplus(1 / X) F$ can be written as elements of $F$.

$$
\begin{aligned}
F_{1} \oplus X F \oplus \frac{1}{X} F & \Longleftrightarrow a_{1} f_{1}+a_{2} f_{2}+X\left(g_{1} f_{1}+g_{2} f_{2}\right)+\frac{1}{X}\left(h_{1} f_{1}+h_{2} f_{2}\right) \\
& \Longleftrightarrow\left(a_{1}+X g_{1}+\frac{1}{X} h_{1}\right) f_{1}+\left(a_{2}+X g_{2}+\frac{1}{X} h_{2}\right) f_{2} \\
& \Longleftrightarrow U f_{1}+V f_{2} \\
& \Longleftrightarrow F(\text { direct sum as } R \text {-module }),
\end{aligned}
$$

where $g_{1}, g_{2}, h_{1}, h_{2}, U$, and $V$ are the elements of $R\left[X, X^{-1}\right]$. Thus with $P_{1}=P \cap F_{1}, P=$ $P_{1} \oplus X F \oplus(1 / X) F$ (direct sum as $R$-module). By Corollary $4.2, F_{1} / P_{1}=F / P$ is a projective over 
$R$, so $F_{1}=P_{1} \oplus P_{1}^{\prime}$ for some $P_{1}^{\prime}$. Now $F=P_{1}\left[X, X^{-1}\right] \oplus P_{1}^{\prime}\left[X, X^{-1}\right]$ and $P=P_{1} \oplus X F \oplus(1 / X) F=P_{1} \oplus$ $X\left(P_{1}\left[X, X^{-1}\right] \oplus P_{1}^{\prime}\left[X, X^{-1}\right]\right) \oplus(1 / X)\left(P_{1}\left[X, X^{-1}\right] \oplus P_{1}^{\prime}\left[X, X^{-1}\right]\right)=P_{1}\left[X, X^{-1}\right] \oplus P_{1}^{\prime}\left[X, X^{-1}\right] \cong F$.

\section{A Qualitative Version of Horrocks' Theorem}

Theorem 5.1. Let $R$ be a local ring and $P$ be a projective $R\left[X, X^{-1}\right]$-module. Then, for any doubly monic polynomial $f$ in $R\left[X, X^{-1}\right]$, the $R\left[X, X^{-1}\right]$-module $P$ and the $R\left[X, X^{-1}\right]_{f}$-module $P_{f}$ have the same minimal number of generators, that is, $\mu(P)=\mu\left(P_{f}\right)$.

Proof. Let $f$ be a doubly monic polynomial in $R\left[X, X^{-1}\right]$. Since there is a natural surjective map $\phi: P \rightarrow P_{f}$, we have $\mu\left(P_{f}\right) \leq \mu(P)$. To complete the proof it is enough to show $\mu\left(P_{f}\right) \geq$ $\mu(P)$. We have the following two cases.

Case 1. $P_{f}$ is a free module over $R\left[X, X^{-1}\right]_{f}$. By Theorem $2.2, P$ is free and the result follows.

Case 2. Suppose $P_{f}$ is not free over $R\left[X, X^{-1}\right]_{f}$. Then $\operatorname{Rank}\left(P_{f}\right)<\mu\left(P_{f}\right)$. Let $\mu\left(P_{f}\right)=n$. We can write $P_{f}$ as a direct summand of $\left(R\left[X, X^{-1}\right]_{f}\right)^{n} \cong\left(R\left[X, X^{-1}\right]^{n}\right)_{f}$. Since $\operatorname{Rank}(P)=\operatorname{Rank}\left(P_{f}\right)<$ $\operatorname{Rank}\left(R\left[X, X^{-1}\right]^{n}\right)$. By Theorem 2.3, there exists an $R\left[X, X^{-1}\right]$-module $P^{\prime}$ such that $P \oplus P^{\prime} \cong$ $R\left[X, X^{-1}\right]^{n}$. Therefore $\mu(P) \leq n=\mu\left(P_{f}\right)$.

\section{References}

[1] D. Hilbert, "Üeber die Theorie der algebraischen Formen," Mathematische Annalen, vol. 36, no. 4, pp. 473-534, 1890.

[2] I. Kaplansky, Homological Dimensions of Rings and Modules, Department of Mathematics, University of Chicago, Chicago, Ill, USA, 1969, reprinted as Part III of Fields and Rings, University of Chicago Press.

[3] D. Eisenbud, Commutative Algebra with a View Toward Algebraic Geometry, vol. 150 of Graduate Texts in Mathematics, Springer, New York, NY, USA, 3rd edition, 1999.

[4] J. P. Serre, Algebre Locale Multiplicites, Mimeographed Notes, vol. 11 of Lecture Notes in Mathematics, Springer, Berlin, Germany, 1965.

[5] M. Auslander and D. A. Buchsbaum, "Codimension and multiplicity," Annals of Mathematics, vol. 68, pp. 625-657, 1958.

[6] M. Auslander and D. A. Buchsbaum, "Homological dimension in local rings," Transactions of the American Mathematical Society, vol. 85, pp. 390-405, 1957.

[7] J. Gago-Vargas, "Bases for projective modules in $A_{n}(k)$, , Journal of Symbolic Computation, vol. 36, no. 6, pp. 845-853, 2003.

[8] R. G. Swan, "A cancellation theorem for projective modules in the metastable range," Inventiones Mathematicae, vol. 27, pp. 23-43, 1974.

[9] G. Horrocks, "Projective modules over an extension of a local ring," Proceedings of the London Mathematical Society, vol. 14, pp. 714-718, 1964.

[10] T. Y. Lam, Serre's Problem on Projective Modules, Springer Monographs in Mathematics, Springer, Berlin, Germany, 2006.

[11] R. A. Rao, "Two examples of the Bass-Quillen-Suslin conjectures," Mathematische Annalen, vol. 279, no. 2, pp. 227-238, 1987.

[12] S. Mandal, "About direct summands of projective modules over Laurent polynomial rings," Proceedings of the American Mathematical Society, vol. 112, no. 4, pp. 915-918, 1991.

[13] B. S. Nashier, "A remark on Horrocks' theorem about projective $A[T]$-modules," Proceedings of the American Mathematical Society, vol. 89, no. 1, pp. 8-10, 1983.

[14] E. Kunz, Introduction to Commutative Algebra and Algebraic Geometry, Birkhäuser, Boston, Mass, USA, 1985.

[15] J. C. Mc Connell and J. C. Robson, Non Commutative Noetherian Rings, John Wiley \& Sons, New York, NY, USA, 1987. 
[16] G.-M. Greuel and G. Pfister, A Singular Introduction to Commutative Algebra, Springer, Berlin, Germany, 2008.

[17] W. Decker, G. M. Greuel, G. Pfister, and H. Schönemann, "Singular 3-1-2-a computer algebra system for polynomial computations," 2010, http://www.singular.uni-kl.de. 


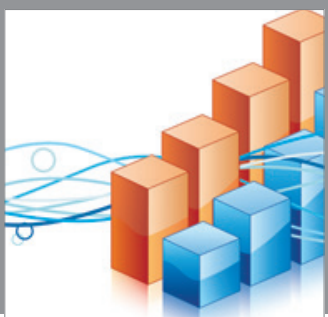

Advances in

Operations Research

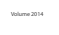

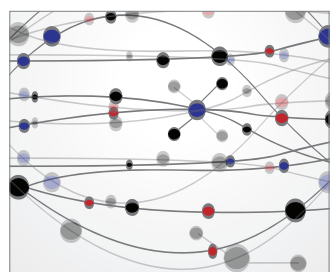

\section{The Scientific} World Journal
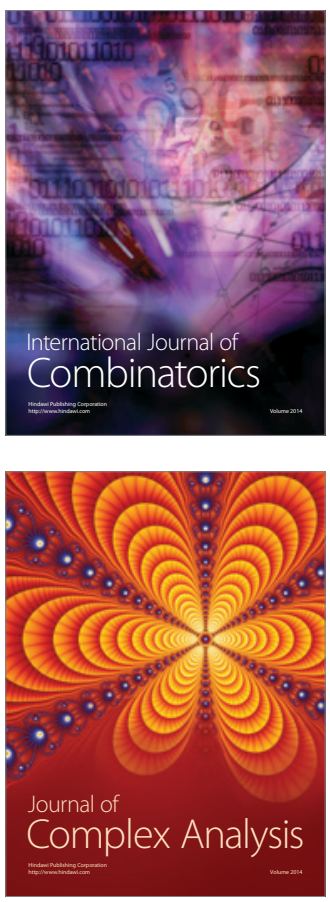

International Journal of

Mathematics and

Mathematical

Sciences
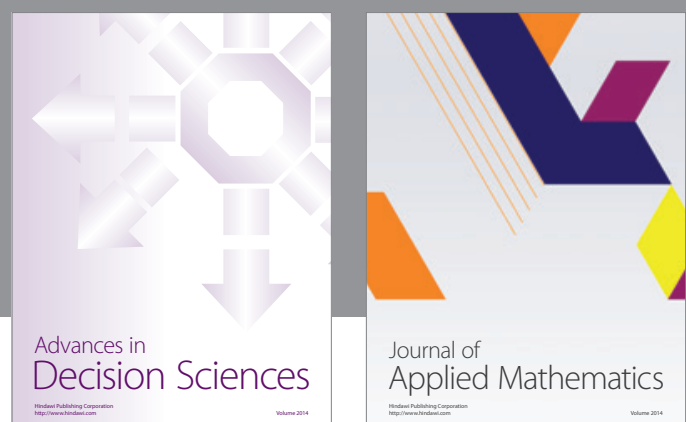

Journal of

Applied Mathematics
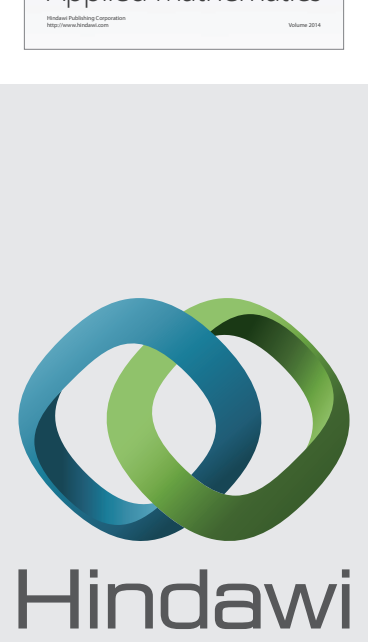

Submit your manuscripts at http://www.hindawi.com
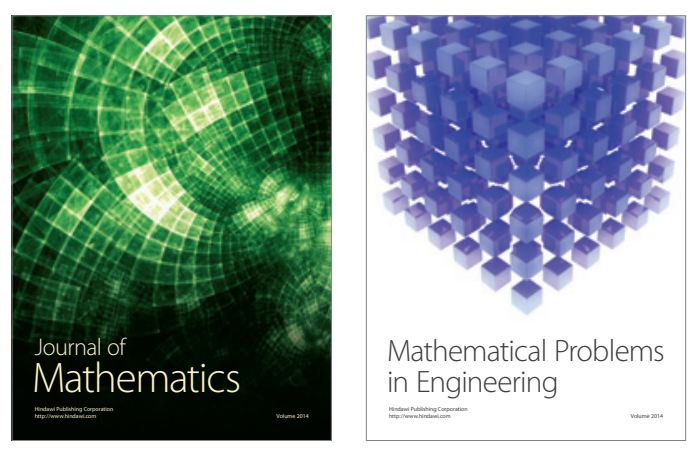

Mathematical Problems in Engineering
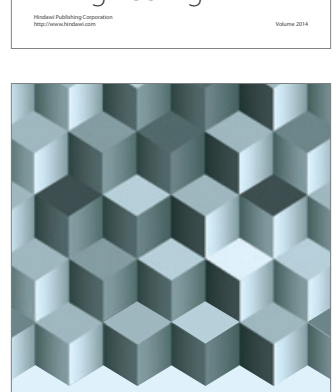

Journal of

Function Spaces
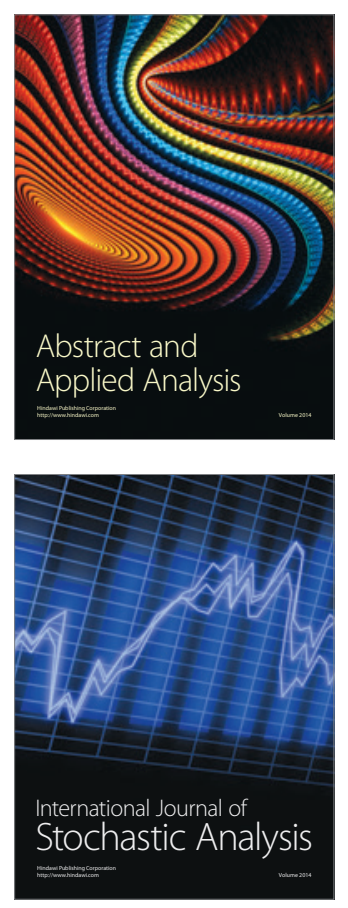

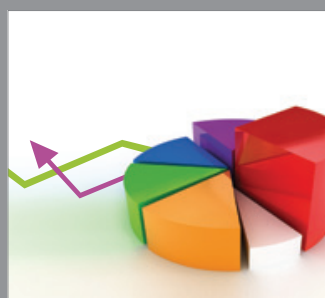

ournal of

Probability and Statistics

Promensencen
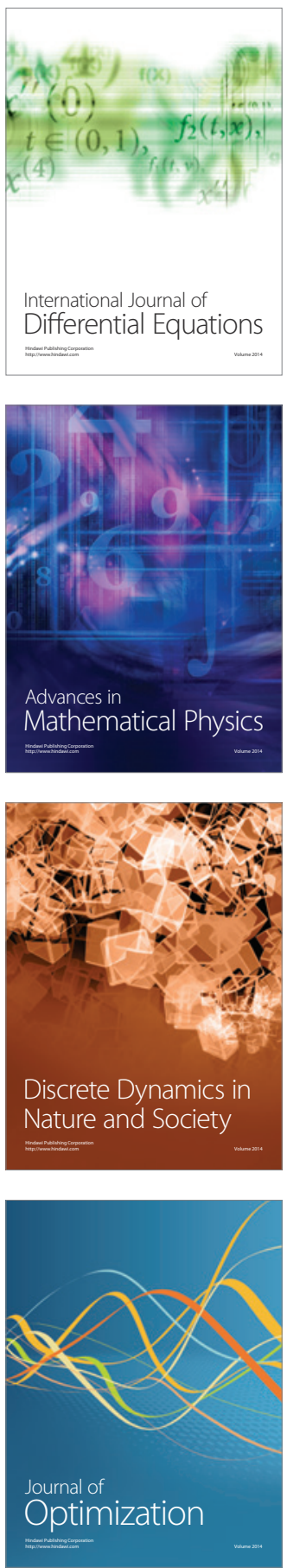